\title{
Treatment of Noncirrhotic Portal Hypertension Secondary to Portal Vein Thrombosis With Endovascular Mesocaval Shunt Creation
}

\author{
Parkpoom Phatharacharukul ${ }^{1}$, Maximilian Pyko ${ }^{2}$, Nabil Fayad ${ }^{1}$ \\ 1. Gastroenterology and Hepatology, Indiana University, Indianapolis, USA 2. Interventional Radiology, Indiana \\ University, Indianapolis, USA
}

Corresponding author: Parkpoom Phatharacharukul, par28034@gmail.com

\begin{abstract}
Transjugular intrahepatic portosystemic shunt (TIPS) creation can be very beneficial to decrease high portal pressure and its consequent dreadful complications, such as variceal hemorrhage. However, some anatomical limitations such as portal vein thrombosis can make TIPS technically impossible to perform. Here, we describe a case of a 72-year-old female patient who previously had a Roux-En-Y choledochojejunostomy, which was complicated by portal vein thrombosis. The patient subsequently developed portal hypertension, and this was successfully treated with endovascular mesocaval shunt creation, given that TIPS was not a viable option.
\end{abstract}

Categories: Radiology, Gastroenterology

Keywords: hemobilia, portal hypertension, portal vein thrombosis, mesocaval shunt, obscure gastrointestinal bleeding

\section{Introduction}

Patients with portal hypertension, both cirrhotic and non-cirrhotic, can develop some serious complications, including variceal hemorrhage and ascites. Treatment modalities include reversing the etiologies of portal hypertension (such as hepatitis $\mathrm{C}$ virus [HCV] eradication in patients with $\mathrm{HCV}$ cirrhosis, or anticoagulation in patients with portal vein thrombosis), directly decreasing the portal vein pressure, and treating the secondary affects (such as diuretics for ascites, or endoscopic banding of esophageal varices).

Received 04/20/2020 Review began 05/02/2020 Review ended 05/07/2020 Published 05/13/2020

() Copyright 2020 Phatharacharukul et al. This is an open access article distributed under the terms of the Creative Commons Attribution License CC-BY 4.0., which permits unrestricted use, distribution, and reproduction in any medium, provided the original author and source are credited.
Creation of a transjugular intrahepatic portosystemic shunt (TIPS), which is an artificial channel connecting the portal and hepatic venous systems, is an effective treatment for portal hypertension [1]. However, TIPS is not always technically feasible due to anatomical inaccessibility, such as in patients with complete portal vein thrombosis.

In these cases, percutaneous mesocaval shunt creation, which connects the mesenteric vessels directly to the systemic circulation through the inferior vena cava, is an alternative treatment technique that can be performed by an experienced interventional radiologist [1].

\section{Case Presentation}

A 72-year-old female with a history of acute necrotizing pancreatitis due to gallstones underwent transgastric pancreatic necrosectomy, which was complicated by recurrent cholangitis secondary to biliary stricture. The patient eventually required a Roux-En-Y choledochojejunostomy for biliary drainage. Her hospital course was complicated by portal vein thrombosis, and she was started on warfarin for anticoagulation. However, four months after surgery, the patient had three recurrent episodes of gastrointestinal bleeding as evidenced by melena and hematochezia. The cause of bleeding was not identified despite multiple investigations including upper endoscopy, push enteroscopy, colonoscopy, video capsule endoscopy, and tagged red blood cell scan. The patient was instructed to stop taking warfarin due to recurrent gastrointestinal bleeding and despite this, she presented to another institution due to bright red blood per rectum. She was transferred to our tertiary care center for further care.

Upon arrival, she was afebrile with a blood pressure of $96 / 49 \mathrm{mmHg}$ and a heart rate of $64 / \mathrm{min}$. Her initial blood tests showed a hemoglobin of $6.8 \mathrm{~g} / \mathrm{dL}$, a platelet of $83,000 / \mathrm{mm}^{3}$, and an international normalized ratio of 1.24. After fluid resuscitation and blood transfusion, the patient underwent a repeat push enteroscopy and colonoscopy without identification of a source of hemorrhage. Due to ongoing bleeding, an antegrade double balloon enteroscopy was performed. The endoscope was traversed through the patient's roux limb and hemobilia emerging from the choledochojejunostomy was visualized (Figure 1). 


\section{Cureus}

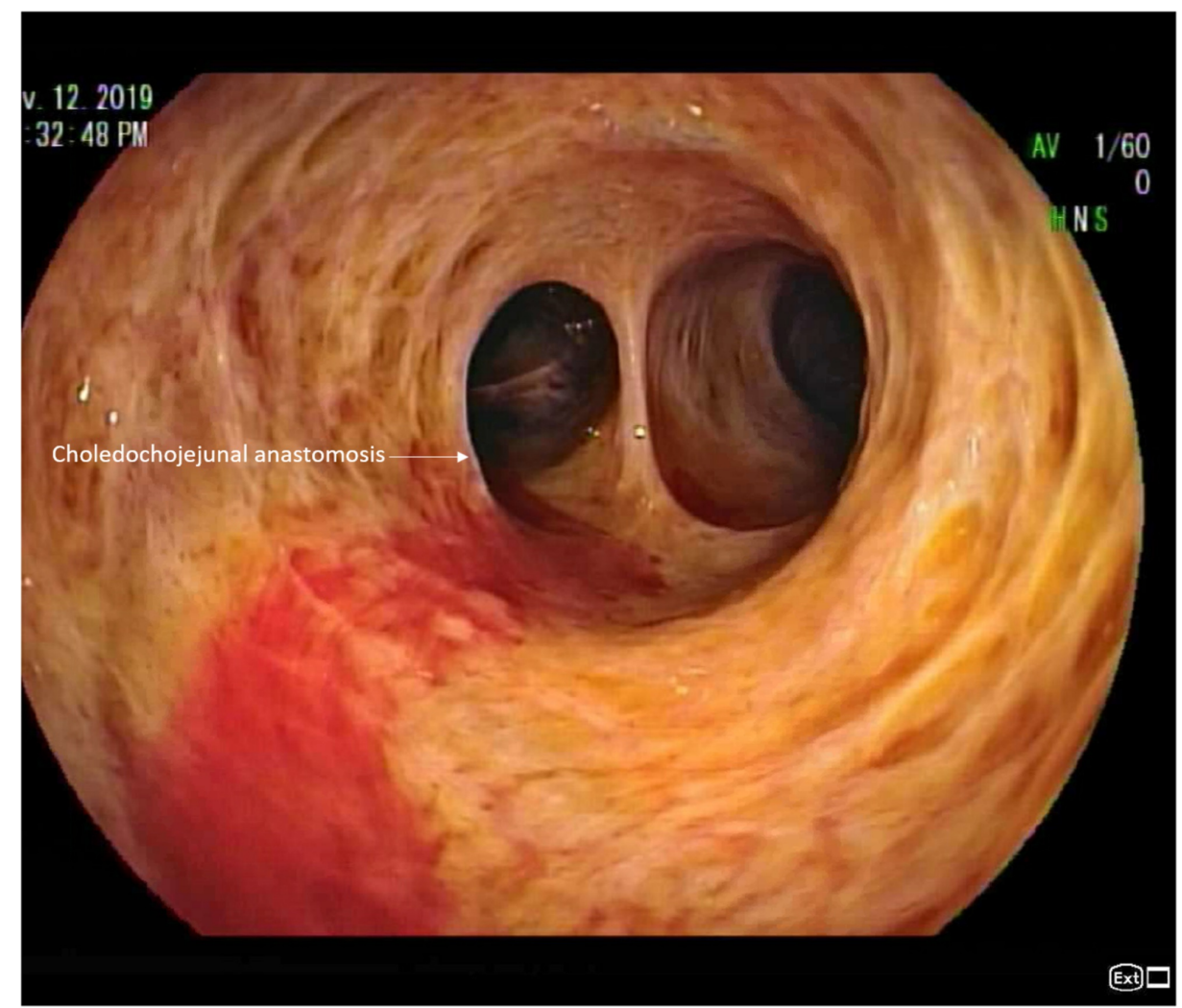

FIGURE 1: Antegrade double baloon enteroscopy shows hemobilia emerging from the choledochojejunostomy

Computed tomography angiography of the abdomen showed portal vein and splenic vein thrombosis, with cavernous transformation and no active bleeding (Figure 2). The source of bleeding was thought to be from periportal venous collateral bleeding. 


\section{Cureus}

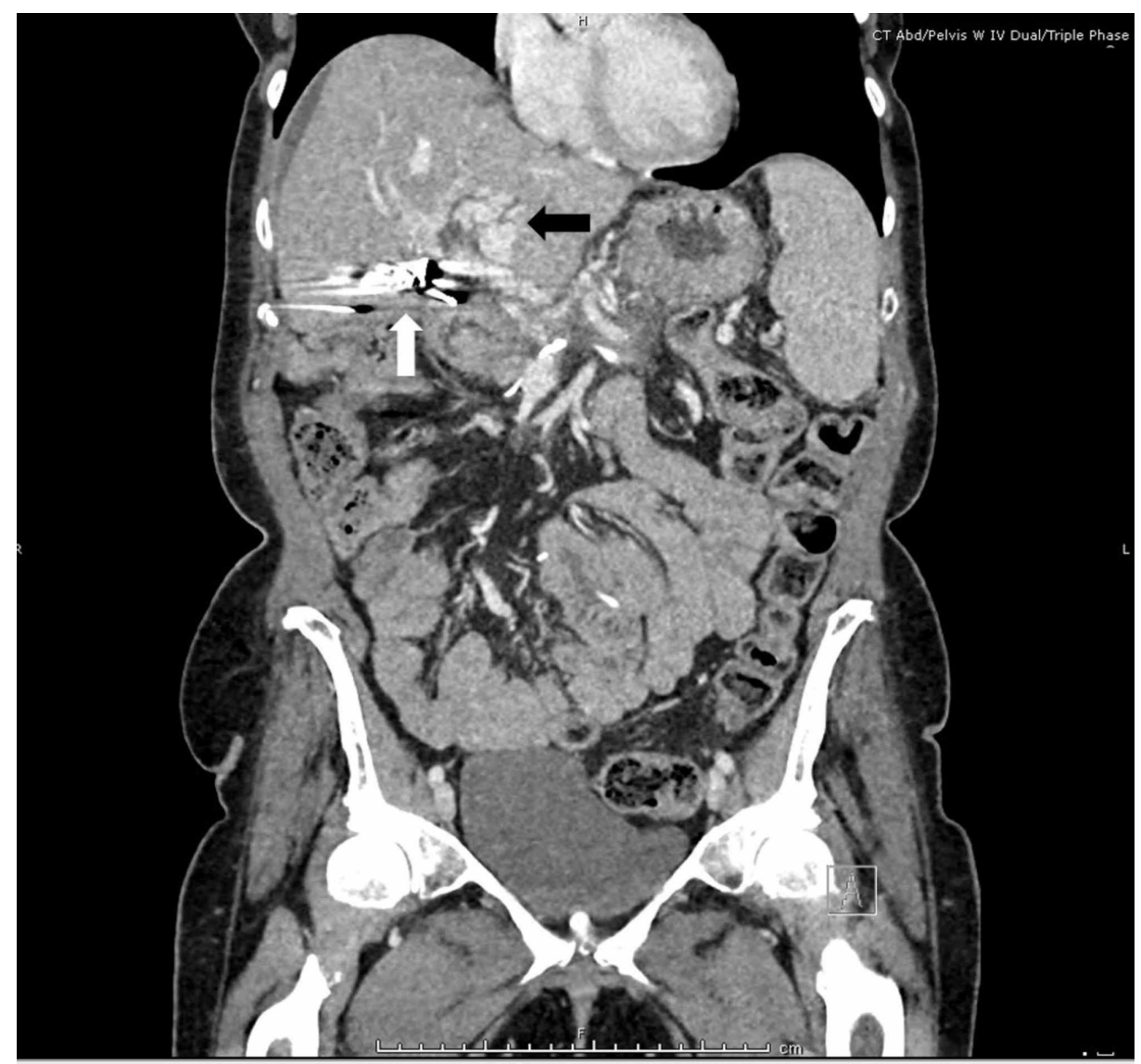

FIGURE 2: Computed tomography angiography of the abdomen and pelvis shows cavernous transformation (black arrow) and metallic artifact from previous surgery (white arrow) with no active bleeding

Interventional radiology was consulted. Initially, portal vein reconstruction with TIPS was considered but was impossible due to complete occlusion of the portal vein with cavernous transformation and lack of a splenic vein to facilitate reconstruction. Thus, endovascular mesocaval shunt was pursued, connecting the inferior vena cava to a varix that extended from the superior mesenteric vein (Figures 3-5).

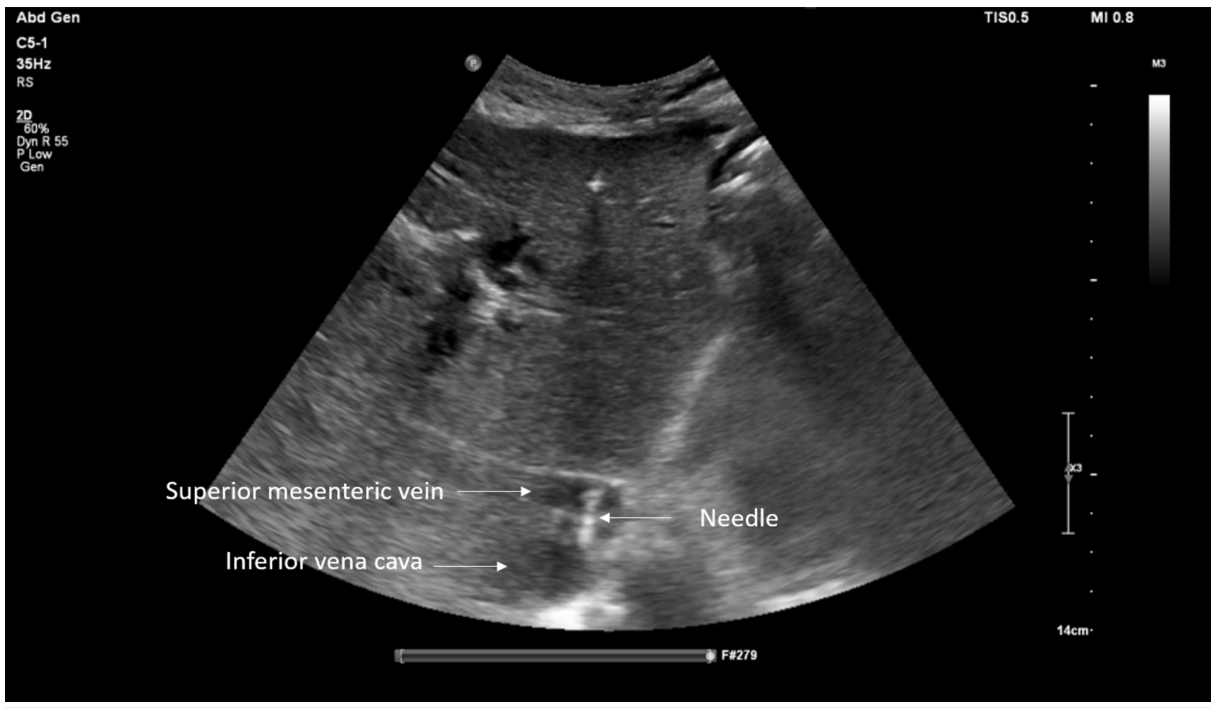

FIGURE 3: Ultrasonographic finding shows the needle catheter system was passed from the inferior vena cava to the cavernous portal vein branch 


\section{Cureus}

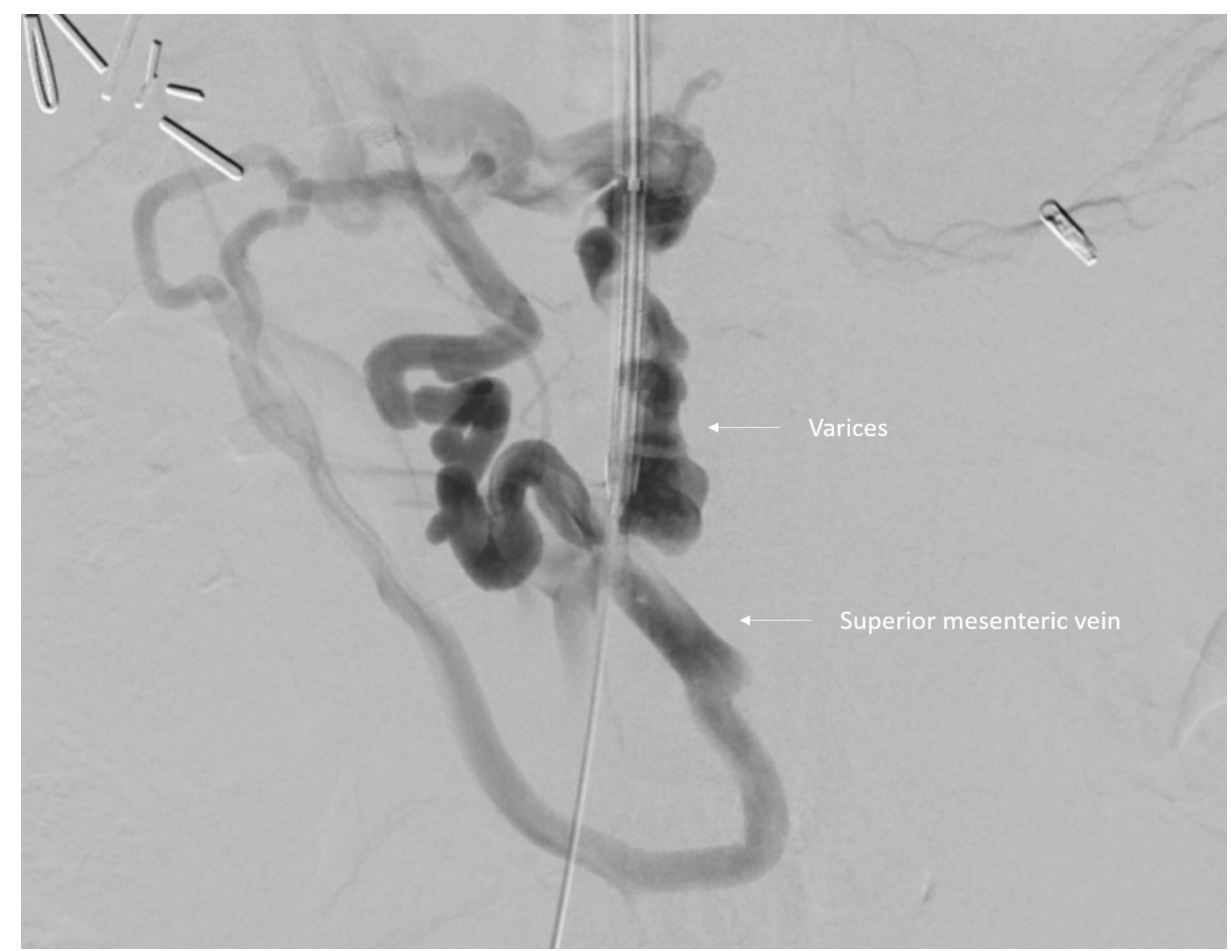

FIGURE 4: Initial venogram shows cavernous transformation with multiple collateral vessels

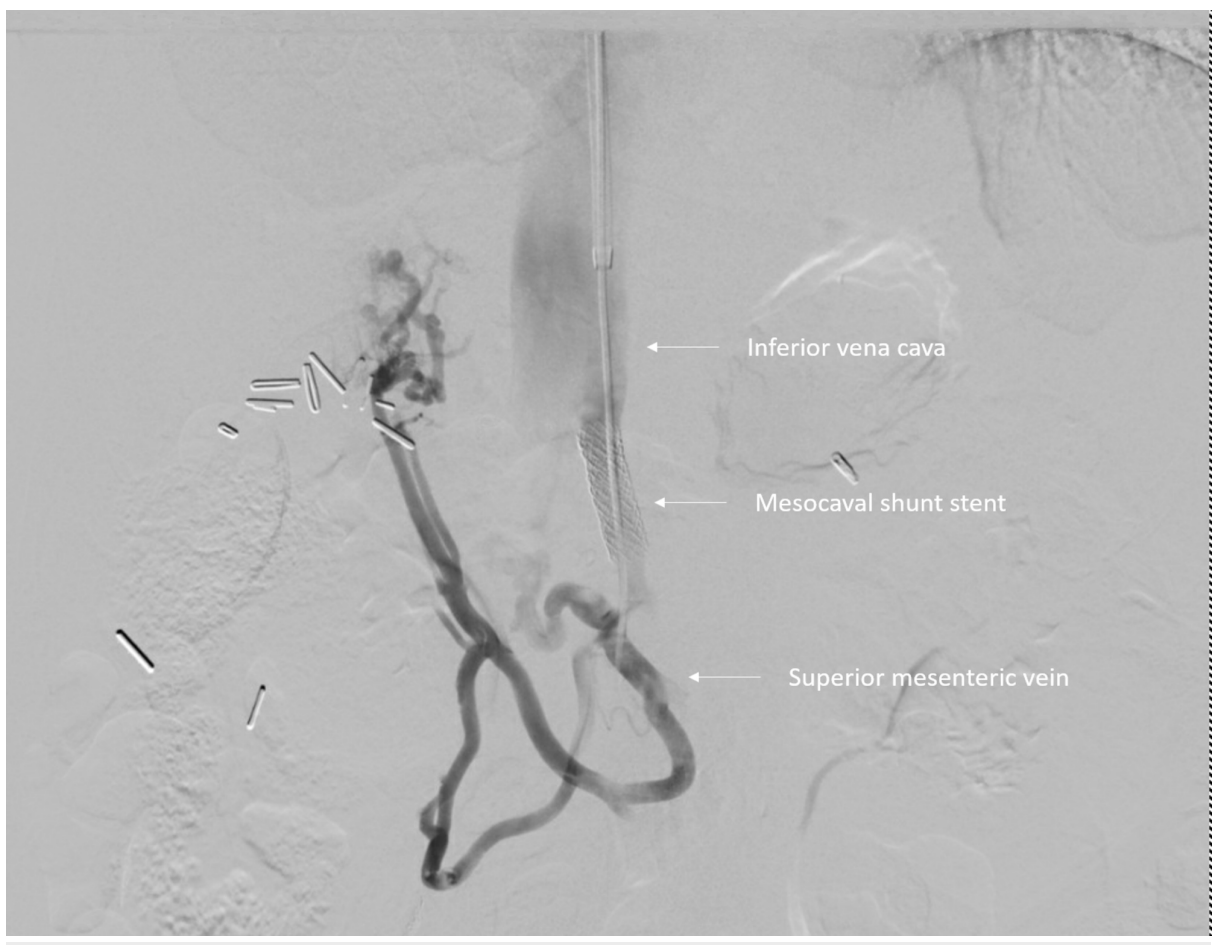

FIGURE 5: Venogram shows a patent mesocaval shunt with blood flowing from the superior mesenteric vein into the inferior vena cava with decreased collateral flow 


\section{Cureus}

The patient did well after the procedure and has not had any recurrent gastrointestinal bleed at three-month follow up. However, she did develop mild hepatic encephalopathy (HE) that is well controlled with lactulose. The presence of mild HE is consistent with a patent shunt, which was also noted on three-month follow-up imaging (Figure 6).

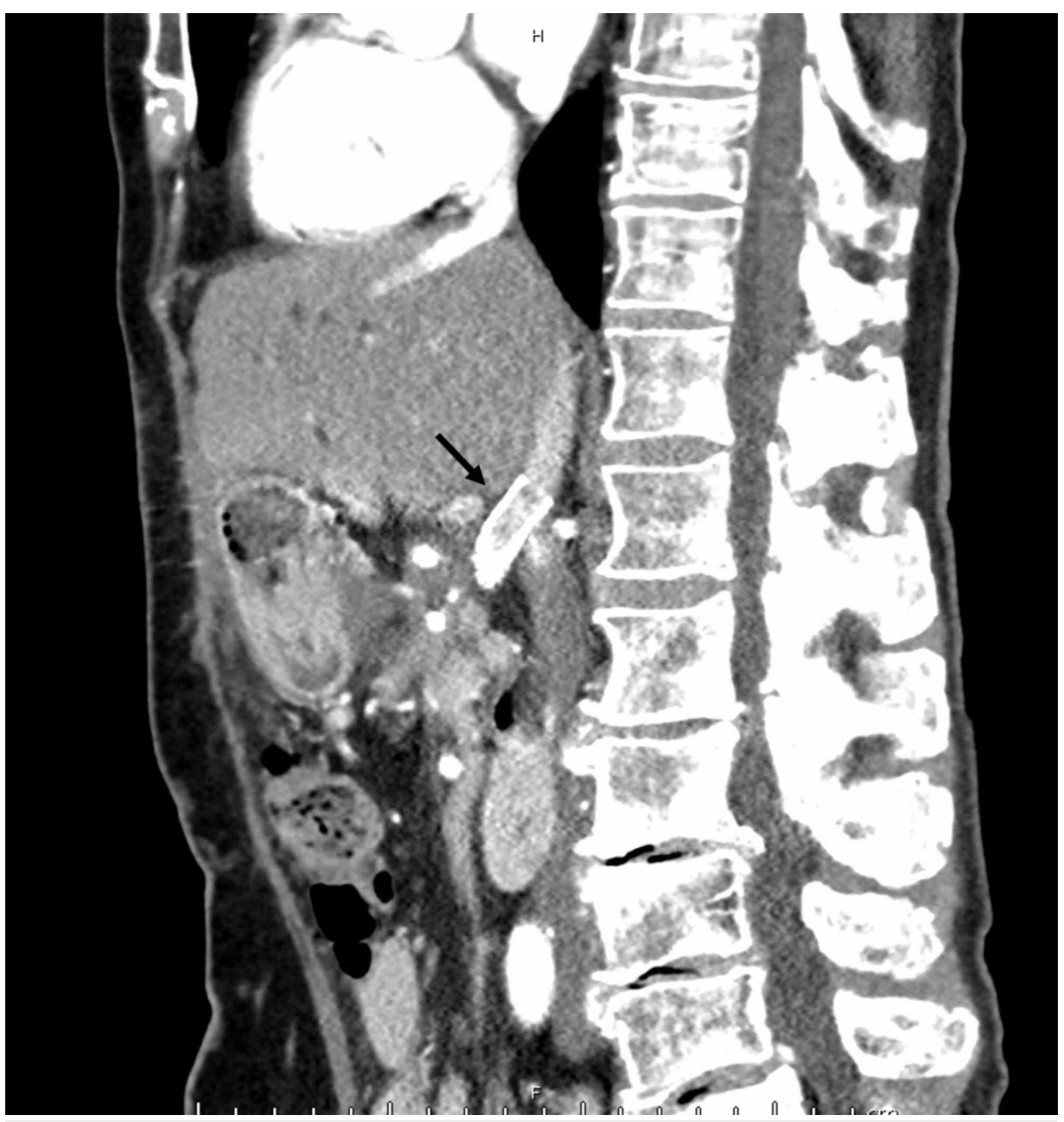

FIGURE 6: Computed tomography of the abdomen with contrast (sagittal view) shows patent shunt with stent (arrow) between superior mesenteric vein and inferior vena cava

\section{Discussion}

Portal hypertension is defined by an increased portal pressure gradient (i.e. the difference in pressure between the portal vein and the inferior vena cava) that is greater than $5 \mathrm{mmHg}$. However, it becomes clinically significant, with consequences such as ascites and variceal bleeding, when the gradient increases to more than $10 \mathrm{mmHg}$ [2]. Without treatment, the outcome is very poor. The mortality in cirrhotic patients complicated by variceal bleeding is up to $57 \%$ at one year [3].

In the absence of contraindications, patients with severe complications of portal hypertension, such as variceal hemorrhage and refractory ascites, should be considered for portal system decompression, including percutaneous shunt placement or surgical shunt. TIPS is a common percutaneous shunt placement procedure for portal hypertension, given long track record and clinical experience [1]. However, there are many absolute and relative contraindications, including congestive heart failure, severe pulmonary hypertension, severe coagulopathy, and portal vein thrombosis, such as in our patient [4].

Mesocaval shunt creation is an alternative option in patients with chronic portal vein occlusion and can be done surgically or percutaneously. It decreases the portal pressure through an artificial channel connecting the mesenteric vessels to the inferior vena cava, thus bypassing the portal vein [5]. The first percutaneous mesocaval shunt was reported in 1996 by Nyman et al. [6]. In addition to overcoming the anatomical 
limitation, it also provides the important benefit of preserving a native portal venous anatomy for subsequent liver transplantation [1].

To be eligible for a mesocaval shunt creation, the patient is required to undergo a contrast-enhanced CT to evaluate for anatomical feasibility. Based on previous case reports, six of the nine patients had no recurrent variceal bleeding after the procedure [7]. However, the strength of evidence is limited due to lack of controlled trials. This procedure should be reserved to experienced interventional radiologists, in order to minimize its risks, including hemorrhage due to puncture of proximal vasculature or other organs [5]. Other complications include HE and subsequent shunt occlusion, which require close follow-ups and repeated imaging [5].

\section{Conclusions}

In patients with portal vein thrombosis and portal hypertension, TIPS procedure might not be possible if the portal veins are completely thrombosed. In such cases, mesocaval shunt creation is one of the treatment options to be considered, and can be done surgically or endovascularly by an experienced interventional radiologist.

\section{Additional Information}

\section{Disclosures}

Human subjects: Consent was obtained by all participants in this study. Conflicts of interest: In compliance with the ICMJE uniform disclosure form, all authors declare the following: Payment/services info: All authors have declared that no financial support was received from any organization for the submitted work. Financial relationships: All authors have declared that they have no financial relationships at present or within the previous three years with any organizations that might have an interest in the submitted work. Other relationships: All authors have declared that there are no other relationships or activities that could appear to have influenced the submitted work.

\section{References}

1. Casadaban LC, Gaba RC: Percutaneous portosystemic shunts: tips and beyond. Semin Intervent Radiol. 2014, 31:227-234. 10.1055/s-0034-1382789

2. Berzigotti A, Seijo S, Reverter E, Bosch J: Assessing portal hypertension in liver diseases . Expert Rev Gastroenterol Hepatol. 2013, 7:141-155. 10.1586/egh.12.83

3. D'Amico G, Garcia-Tsao G, Pagliaro L: Natural history and prognostic indicators of survival in cirrhosis: a systematic review of 118 studies. J Hepatol. 2006, 44:217-231. 10.1016/j.jhep.2005.10.013

4. Boyer TD, Haskal ZJ: The role of transjugular intrahepatic portosystemic shunt (TIPS) in the management of portal hypertension: update 2009. Hepatology. 2010, 51:306. 10.1002/hep.23383

5. Davis J, Chun AK, Borum ML: Could there be light at the end of the tunnel? mesocaval shunting for refractory esophageal varices in patients with contraindications to transjugular intrahepatic portosystemic shunt. World J Hepatol. 2016, 8:790-795. 10.4254/wjh.v8.i19.790

6. Nyman URO, Semba CP, Chang H, Hoffman C, Dake MD: Percutaneous creation of a mesocaval shunt . J Vasc Interv Radiol. 1996, 7:769-773. 10.1016/s1051-0443(96)70847-3

7. Yoon JK, Kim MD, Lee DY, Han SJ: Mesocaval shunt creation for jejunal variceal bleeding with chronic portal vein thrombosis. Yonsei Med J. 2018, 59:162-166. 10.3349/ymj.2018.59.1.162 